\title{
Synthesis of a sucrose-based macrocycle with unsymmetrical monosaccharides "arms"
}

\author{
Karolina Tiara, Mykhaylo A. Potopnyk and Sławomir Jarosz ${ }^{*}$
}

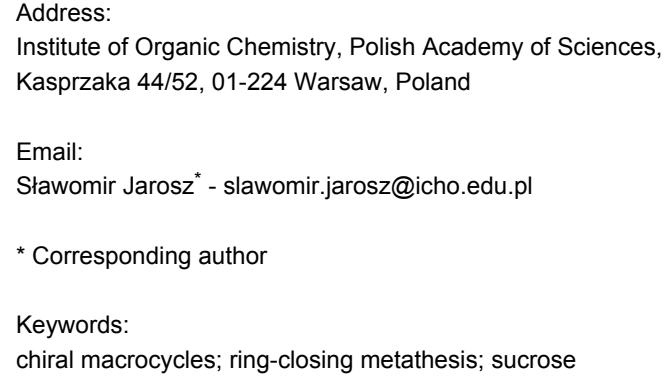

Beilstein J. Org. Chem. 2018, 14, 634-641. doi:10.3762/bjoc. 14.50

Received: 04 January 2018

Accepted: 21 February 2018

Published: 15 March 2018

Associate Editor: N. Sewald

() 2018 Tiara et al.; licensee Beilstein-Institut. License and terms: see end of document.

\begin{abstract}
An efficient methodology for the selective substitution of both terminal positions (C6 and C6') in 1',2,3,3',4,4'-hexa- $O$-benzylsucrose with different unsaturated monosaccharide units is presented. Such a highly functionalized intermediate was cyclized under RCM conditions to afford a macrocyclic derivative containing a 31-membered ring in $26 \%$ yield.
\end{abstract}

\section{Introduction}

Chiral macrocyclic compounds play an important role in supramolecular and biological systems [1,2]. Many of them serve as convenient receptors for cations [3], anions [4], ion pairs [5], neutral molecules [6] etc.

Binaphthols [7-9], amino acids [10], chiral diamines [11,12], carbohydrates [13], etc. are usually applied as building blocks for construction of such type of compounds.

We are engaged in the synthesis of such macrocyclic derivatives containing the most common natural disaccharide, sucrose $[14,15]$. Several different classes of macrocyclic derivatives, including: crown [16] and aza-crown [17,18] derivatives, macrocyclic dilactams [19,20], and ureas [21], were prepared in our laboratory.
Sucrose was also used by other groups as a precursor for the preparation of biodegradable polymers [22-24] and polymeric nanoparticles [25]. On the other hand, sucrose derivatives demonstrate antimicrobial and antitumor activities [26,27].

\section{Results and Discussion}

Recently, we have prepared sucrose-based macrocyclic derivative 4 in which the terminal positions of this disaccharide (C6 and C6') are connected via a long polyhydroxylated bridge [28]. In this model study, both terminal positions in 6,6'-diamino1',2,3,3',4,4'-hexa-O-benzyl-6,6'-dideoxysucrose (2) were elongated with the same polyhydroxylated unit $\mathbf{1}$ providing diamide $\mathbf{3}$, which subsequently underwent cyclization under the chosen ring-closing metathesis (RCM) conditions [29,30] to give the 21-membered macrocycle 4 (Scheme 1). 


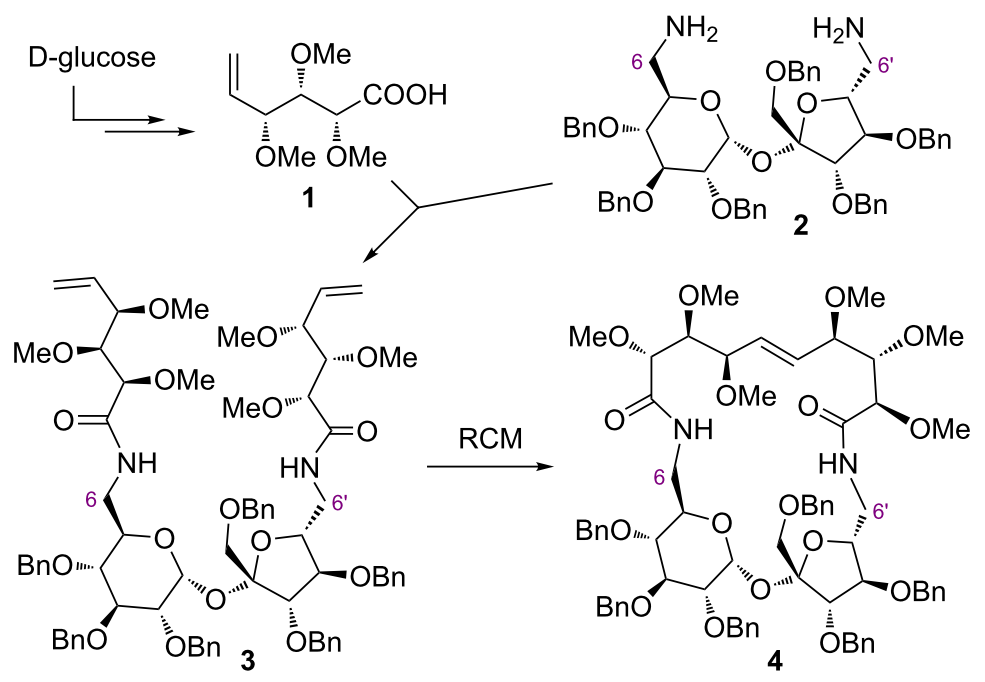

Scheme 1: Synthesis of macrocyclic derivative 4

The reduction of the amide functions should lead to amines, which might be used as starting materials for the preparation of, e.g., cryptands 6 (Figure 1). All attempts, however, to reduce 4 to diamine $\mathbf{5}$ were unsuccessful.
We have decided, therefore, to elaborate another method leading to functionalized sucrose amines of type 9 (Figure 2) which will be obtained by a selective introduction of different fragments 8 (obtained from, e.g., glucose, mannose, etc.).

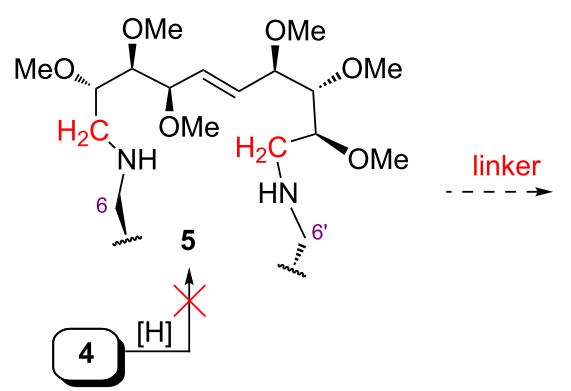

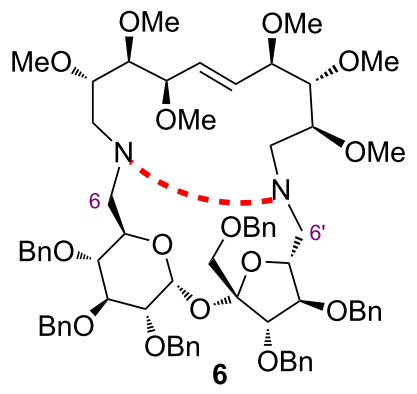

Figure 1: Possible route to sucrose cryptands 6.

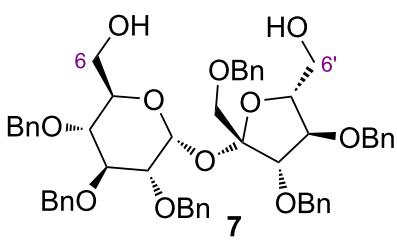<smiles>C=CC(OC)C(OC)=C(CN)COC</smiles>

selective introduction

Figure 2: Possible route to dienes of type 9. 
At this stage we were focused on the elaboration of a methodology allowing to introduce different fragments at the sucrose terminals. We chose, therefore, derivatives of methylated hexitols which are easy to prepare and are more convenient than benzyls in the interpretation of the NMR spectra.

We faced, however, a serious problem in the synthesis of amines of type $\mathbf{8}$. Treatment of aldehyde $\mathbf{1 1}$ - generated in situ from iodide $\mathbf{1 0}$ according to Vasellas' procedure [31,32] - with benzylamine under the reductive amination conditions afforded an inseparable mixture of two products differing in the configuration at the $\mathrm{C} 2$ center (12a and $\mathbf{1 2 b}$; Scheme 2); such a phenomenon - epimerization under these conditions - is known [33].

The alternative way to the desired amine 12a, based on the $\mathrm{S}_{\mathrm{N}} 2$ reaction of the activated alcohol $\mathbf{1 3}$ [34,35] with benzylamine, also failed (Scheme 2).
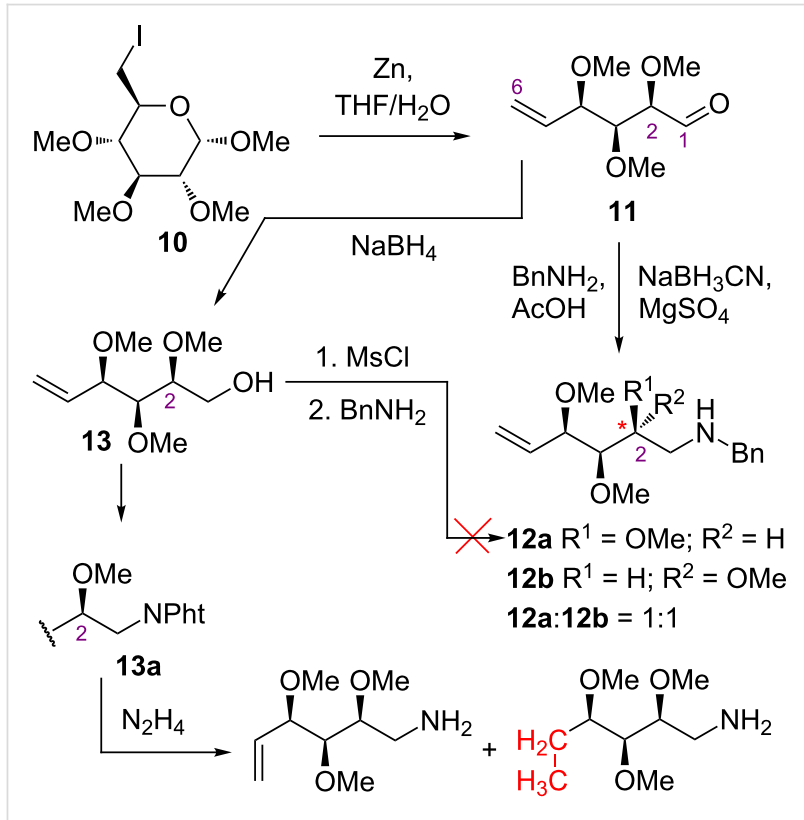

13b $\mathrm{m} / \mathrm{z}=189 \quad$ 13c $\mathrm{m} / \mathrm{z}=191$

Scheme 2: Unsuccessful attempts to amines 12a and 13b.

The Mitsunobu approach to convert the hydroxy group into an amine function was also unsuccessful. Although 13 reacted with phthalimide gave the desired product 13a, the deprotection of the amine function with hydrazine caused also reduction of the C5-C6 double bond; two peaks at 189 and 191 Da were observed in the MS spectrum of crude post-reaction mixture (for 13b and 13c, respectively).

We reasoned, that the all these problems may be overcome by an elongation of alcohol $\mathbf{1 3}$ (derived from D-glucose) with a rigid fragment and we decided to introduce the phenyl ring. Treatment of alcohol 13 with para-nitrophenol under Mitsunobu conditions afforded the nitro compound 14 in $63 \%$ yield. Stereoisomeric alditol 15, obtained from D-mannose, was converted analogously to $\mathbf{1 6}$ (in $60 \%$ yield). Both nitro compounds 14 and 16 were reduced to the corresponding amines 17 and $\mathbf{1 8}$ with sodium dithionite (Scheme 3).
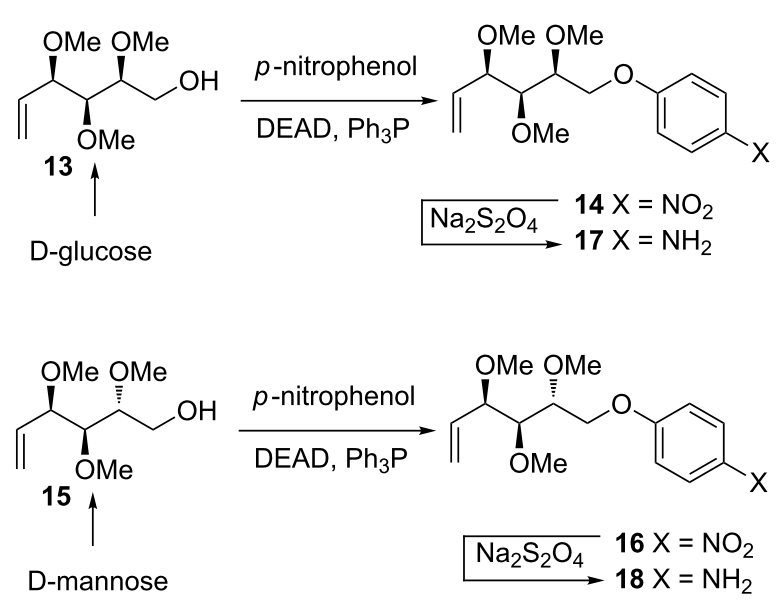

Scheme 3: Syntheses of "elongated" amines 17 and 18.

The synthesis of the macrocycle with different arms at both terminal positions was initiated from 6'-O-tert-butyldiphenylsilyl1',2,3,3',4,4'-hexa- $O$-benzylsucrose (19) readily available by a selective silylation of $1^{\prime}, 2,3,3^{\prime}, 4,4^{\prime}$-hexa- $O$-benzylsucrose (7) [36].

Aldehyde 20 [37] - obtained by Swern oxidation [38] of alcohol 19 - was reacted with amine $\mathbf{1 7}$ to afford the desired amine isolated as acetate $\mathbf{2 1}$ in $85 \%$ total yield. Removal of the TBDPS protecting group from the C6'-position gave alcohol 22 in $97 \%$ yield. Under the same "Swern oxidation-reductive amination-acetylation" conditions, alcohol 22 was converted into aldehyde 23, which reacted further with amine $\mathbf{1 8}$, furnishing diolefin 24 in $64 \%$ total yield. Cyclization of precursor 24 induced by the Hoveyda-Grubbs catalyst (II gen.) afforded the target macrocycle 25 in $26 \%$ yield (Scheme 4 ). The $E$-configuration of the newly created $\mathrm{C}=\mathrm{C}$-bond in the final product was proven by ${ }^{1} \mathrm{H}$ NMR analysis $\left(J_{15-15},=15.9 \mathrm{~Hz}\right)$.

\section{Conclusion}

In summary, we proposed an efficient method of the synthesis of a 31-membered macrocycle with sucrose scaffold. The proposed methodology allows for the regioselective introduction of various polyhydroxylated unsaturated fragments (derived from different sugars) at either terminal position of sucrose which 

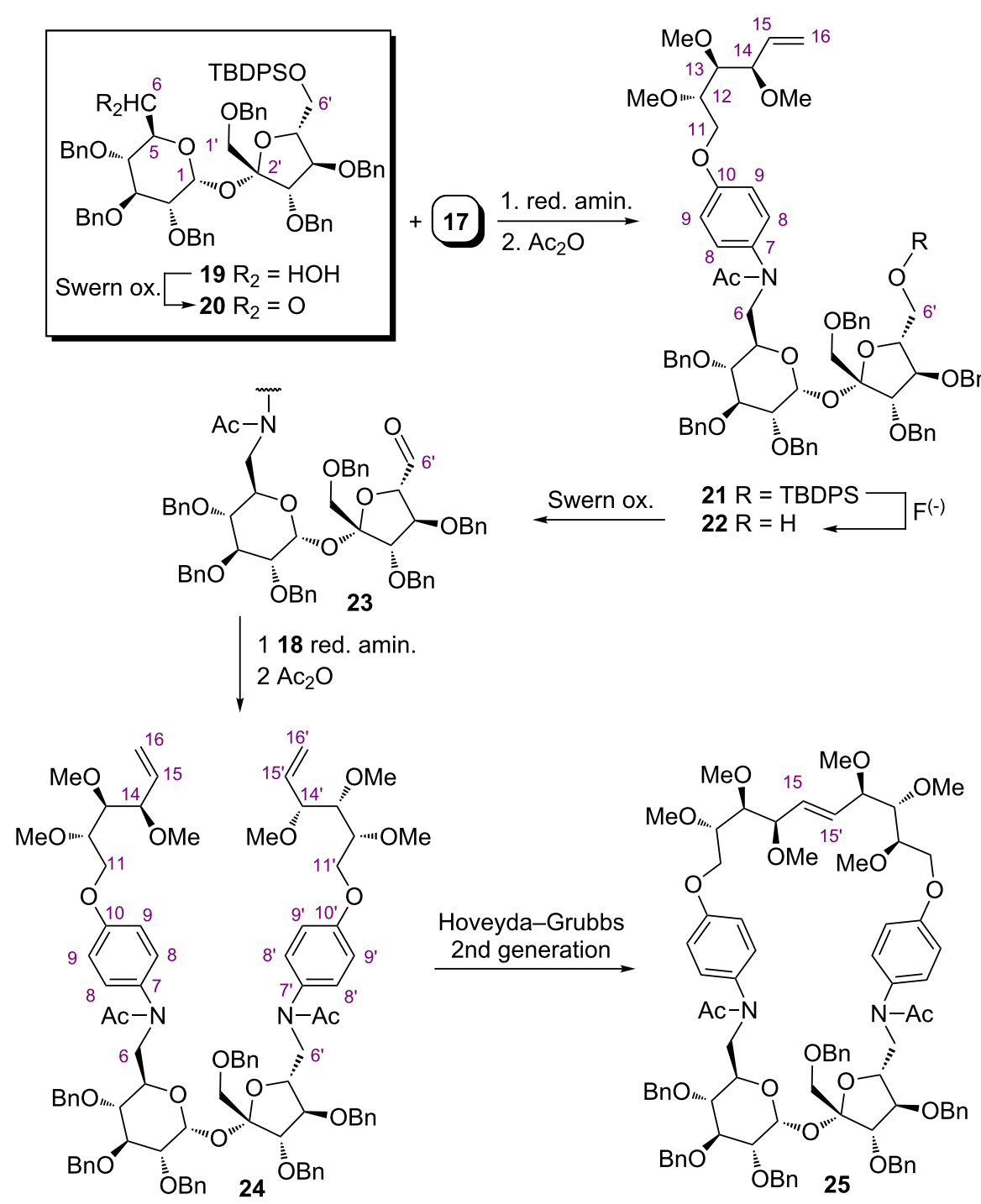

Scheme 4: Synthesis of macrocycle 25.

undergo an efficient cyclization under the RCM conditions. Although, for practical reasons, the method was elaborated for the methylated derivatives of hextitol pendants it might be, eventually, applied also for synthons with other protecting groups.

\section{Experimental}

\section{General}

The NMR spectra were recorded with a Varian VNMRS $600 \mathrm{MHz}$ spectrometer for solutions in $\mathrm{CDCl}_{3}$ at $25^{\circ} \mathrm{C}$. The ${ }^{13} \mathrm{C}$ NMR data for compound 25 were recorded with a Varian VNMRS $500 \mathrm{MHz}$ spectrometer. The structures were assigned, whenever necessary, with the help of $2 \mathrm{D}$ correlation experiments (COSY, HSQC, HMBC). Chemical shifts were reported with reference to TMS. Optical rotations were measured with a Jasco P 1020 polarimeter (sodium light) in chloroform at room temperature. Mass spectra were recorded with a Synapt G2-S HDMS (Waters Inc) mass spectrometer equipped with an electrospray ion source and a q-TOF type mass analyzer. The instrument was controlled and recorded data were processed using the MassLynx V4.1 software package (Waters Inc). Thinlayer chromatography (TLC) was performed on silica gel plates coated with fluorescent indicator. Column chromatography was performed on silica gel (Merck, 230-400 mesh). Organic solutions were dried over anhydrous $\mathrm{MgSO}_{4}$.

\section{Procedure for the synthesis of nitro com- pounds 14 and 16}

To a solution of alcohol 13 (310 mg, $1.63 \mathrm{mmol})$ in dry THF $(12 \mathrm{~mL})$ and toluene $(4 \mathrm{~mL}), \mathrm{Ph}_{3} \mathrm{P}(1.28 \mathrm{~g}, 4.89 \mathrm{mmol})$ and para-nitrophenol (340 mg, $2.44 \mathrm{mmol}$ ) were added. After stir- 
ring during $15 \mathrm{~min}$, DEAD $(384 \mu \mathrm{L}, 2.44 \mathrm{mmol})$ was added dropwise. The reaction mixture was stirred for $3 \mathrm{~h}$ at room temperature and then partitioned between diethyl ether $(20 \mathrm{~mL})$ and $5 \% \mathrm{NaOH}$ aqueous solution $(30 \mathrm{~mL})$. The layers were separated and the aqueous one extracted with diethyl ether $(3 \times 15 \mathrm{~mL})$. The combined organic solutions were washed with water $(15 \mathrm{~mL})$ and brine $(15 \mathrm{~mL})$, dried, concentrated, and the resulting residue was purified by flash chromatography (hexanes-ethyl acetate, 70:30) to afford pure product 15 (318 $\mathrm{mg}, 1.02 \mathrm{mmol}, 63 \%)$ as a white solid. TLC [hexanes-AcOEt (2:1)]: $R_{\mathrm{f}}=0.3 ;[\alpha]_{\mathrm{D}}{ }^{22}+28.5 ;{ }^{1} \mathrm{H}$ NMR $\delta 8.18$ (d, $J=9.3 \mathrm{~Hz}, 2 \mathrm{H}, \mathrm{ArH}), 6.99$ (d, $J=9.3 \mathrm{~Hz}, 2 \mathrm{H}, \operatorname{ArH}), 6.03$ (ddd, $\left.J_{5,4}=6.0 \mathrm{~Hz}, J_{5,6}=10.7 \mathrm{~Hz}, J_{5.6}=17.3 \mathrm{~Hz}, 1 \mathrm{H}, \mathrm{H}-5\right)$, 5.39 (m, 2H, H-6, H-6'), 4.95 (dd, $J_{4,5}=6.2 \mathrm{~Hz}, J_{4,3}=7.3 \mathrm{~Hz}$, $1 \mathrm{H}, \mathrm{H}-4), 3.63-3.54(\mathrm{~m}, 3 \mathrm{H}, 2 \times \mathrm{H}-1, \mathrm{H}-2), 3.53$ (dd, $J_{3,2}=2.5$ $\mathrm{Hz}, 1 \mathrm{H}, \mathrm{H}-3), 3.49$ (s, 3H, OMe), 3.42 (s, 3H, OMe), 3.29 (s, $3 \mathrm{H}, \mathrm{OMe}) \mathrm{ppm} ;{ }^{13} \mathrm{C}$ NMR $\delta 162.73$ (C-Ar), 141.57 (C-Ar), 133.98 (C-5), 125.82 (2 × C-Ar), 119.07 (C-6), $115.51(2 \times$ C-Ar), 82.30 (C-3), 78.61 (C-2), 77.54 (C-4), 70.74 (C-1), 61.20 (OMe), 59.09 (OMe), 59.08 (OMe) ppm; HRMS (ESI) $[\mathrm{M}+\mathrm{Na}]^{+}$: calcd for $\mathrm{C}_{15} \mathrm{H}_{21} \mathrm{NO}_{6} \mathrm{Na}, 334.1257$; found, 334.1256; anal. calcd for $\mathrm{C}_{15} \mathrm{H}_{21} \mathrm{NO}_{6}$ (311.33): C, 57.87; $\mathrm{H}$, $6.80 ; \mathrm{N}, 4.50$; found: $\mathrm{C}, 57.65 ; \mathrm{H}, 6.79 ; \mathrm{N}, 4.57$.

Nitro compound 16 was obtained as a white solid in $60 \%$ yield (295 mg, $0.95 \mathrm{mmol}$ ), using the same procedure, from alcohol 15 (300 mg, $1.58 \mathrm{mmol}), \mathrm{Ph}_{3} \mathrm{P}$ (1.24 g, $4.73 \mathrm{mmol}$ ), para-nitrophenol (329 mg, $2.37 \mathrm{mmol})$, and DEAD (371 $\mu \mathrm{L}, 2.37 \mathrm{mmol})$. TLC [hexanes-AcOEt (2:1)]: $R_{\mathrm{f}}=0.3 .[\alpha]_{\mathrm{D}}{ }^{22}-18.9 ;{ }^{1} \mathrm{H}$ NMR $\delta$ 8.21 (d, $J=10 \mathrm{~Hz}, 2 \mathrm{H}, \mathrm{ArH}), 7.03$ (d, $J=10 \mathrm{~Hz}, 2 \mathrm{H}, \mathrm{ArH})$, $5.92\left(\mathrm{ddd}, J_{5,4}=7.8 \mathrm{~Hz}, J_{5,6}=10.4 \mathrm{~Hz}, J_{5.6}=17.3 \mathrm{~Hz}, 1 \mathrm{H}\right.$, H-5), 5.41-5.34 (m, 2H, $2 \times \mathrm{H}-6), 4.39$ (dd, $J_{1,1}=10.3 \mathrm{~Hz}, J_{1,2}$ $=2.3 \mathrm{~Hz}, 1 \mathrm{H}, \mathrm{H}-1), 4.19\left(\mathrm{dd}, J_{1,1}=10.3 \mathrm{~Hz}, J_{1,2}=4.7 \mathrm{~Hz}, 1 \mathrm{H}\right.$, $\mathrm{H}-1), 3.86$ (m, 1H, H-4), 3.75 (m, 1H, H-2), 3.49 (s, 3H, OMe), 3.43 (m, 4H, OMe, H-3), 3.34 (s, 3H, OMe) ppm; ${ }^{13} \mathrm{C}$ NMR $\delta$ 163.82 (C-Ar), 141.61 (C-Ar), 135.54 (C-5), 125.89 ( $2 \times \mathrm{C}-\mathrm{Ar})$, $118.71(\mathrm{C}-6), 114.58(2 \times \mathrm{C}-\mathrm{Ar}), 82.59(\mathrm{C}-3), 81.81(\mathrm{C}-4)$, $78.81(\mathrm{C}-2), 67.56(\mathrm{C}-1), 61.25,58.45,56.72(3 \times \mathrm{OMe}) \mathrm{ppm}$; HRMS (ESI) $[\mathrm{M}+\mathrm{Na}]^{+}$: calcd for $\mathrm{C}_{15} \mathrm{H}_{21} \mathrm{NO}_{6} \mathrm{Na} 334.1257$; found: 334.1256; anal. calcd for $\mathrm{C}_{15} \mathrm{H}_{21} \mathrm{NO}_{6}$ (311.33): $\mathrm{C}$, 57.87; H, 6.80; N, 4.50; found: C, 57.69; H, 6.81; N, 4.50 .

\section{Synthesis of amino compounds 17 and 18}

To a solution of nitro compound $\mathbf{1 4}$ or $\mathbf{1 6}$ (232 $\mathrm{mg}, 0.74 \mathrm{mmol})$ in aq ethanol (14 mL, 1:1 v/v), $\mathrm{K}_{2} \mathrm{CO}_{3}(304 \mathrm{mg}, 2.22 \mathrm{mmol})$ and $\mathrm{Na}_{2} \mathrm{~S}_{2} \mathrm{O}_{4}(322 \mathrm{mg}, 1.85 \mathrm{mmol}$ ) were added, and the mixture was stirred for $30 \mathrm{~min}$ at rt. Ethyl acetate $(15 \mathrm{~mL})$ was added, the layers were separated, and the aqueous one extracted with ethyl acetate $(3 \times 8 \mathrm{~mL})$. The combined organic solutions were dried, concentrated, and the crude product $\mathbf{1 7}$ or $\mathbf{1 8}$ was used in the next step without further purification.

\section{Synthesis of compound $\mathbf{2 1}$}

A solution of amine $17(203 \mathrm{mg}, 0.72 \mathrm{mmol})$ in DCM (10 mL) was added to a solution of aldehyde $\mathbf{2 0}(202 \mathrm{mg}, 0.18 \mathrm{mmol}$; prepared from alcohol 19 as described in our previous paper [37]) in DCM $(10 \mathrm{~mL})$ containing acetic acid $(41 \mu \mathrm{L}$, $0.72 \mathrm{mmol})$ and $\mathrm{MgSO}_{4}(\approx 200 \mathrm{mg})$, and the mixture was stirred for $1 \mathrm{~h}$ at rt. Then, $\mathrm{NaBH}_{3} \mathrm{CN}(17 \mathrm{mg}, 0.72 \mathrm{mmol})$ was added and stirring was continued overnight. Water $(20 \mathrm{~mL}), 0.1 \mathrm{M}$ solution of $\mathrm{NH}_{3}(2 \mathrm{~mL})$, and DCM $(15 \mathrm{~mL})$ were added, the layers were separated, and the aqueous one extracted with DCM $(3 \times 10 \mathrm{~mL})$. The combined organic solutions were washed with water $(10 \mathrm{~mL})$ and brine $(10 \mathrm{~mL})$, dried and concentrated. The residue was dissolved in 1,4-dioxane $(10 \mathrm{~mL})$ to which DMAP (4 mg, $0.04 \mathrm{mmol}), \mathrm{Et}_{3} \mathrm{~N}(126 \mu \mathrm{L}, 0.90 \mathrm{mmol})$, and acetic anhydride $(51 \mu \mathrm{L}, 0.54 \mathrm{mmol})$ were added and the mixture was stirred overnight at $100{ }^{\circ} \mathrm{C}$. After cooling to rt, water $(15 \mathrm{~mL})$ and DCM $(15 \mathrm{~mL})$ were added, the layers were separated, and the aqueous one was extracted with DCM $(3 \times 10 \mathrm{~mL})$. Combined organic solutions were washed with water $(10 \mathrm{~mL})$ and brine $(10 \mathrm{~mL})$, dried, concentrated, and the crude productwas purified by flash chromatography (hexanes-ethyl acetate, 80:20 to 50:50) to afford 21 (219 $\mathrm{mg}, 0.15 \mathrm{mmol}, 85 \%)$ as a colorless oil. TLC [hexanes-AcOEt $(2: 1)]: R_{\mathrm{f}}=0.2 ;[\alpha]_{\mathrm{D}}{ }^{22}$ $+13.6 ;{ }^{1} \mathrm{H}$ NMR $\delta 7.66(\mathrm{~m}, 4 \mathrm{H}, \mathrm{ArH}), 7.33-7.15(\mathrm{~m}, 36 \mathrm{H}$, $\operatorname{ArH}), 7.05\left(\mathrm{~d}, J_{9,8}=8.7 \mathrm{~Hz}, 2 \mathrm{H}, 2 \times \mathrm{H}-9\right), 6.77\left(\mathrm{~d}, J_{8,9}=8.7\right.$ $\mathrm{Hz}, 2 \mathrm{H}, 2 \times \mathrm{H}-8), 5.92\left(\mathrm{ddd}, J_{15,14}=6.0 \mathrm{~Hz}, J_{15,16}=10.6 \mathrm{~Hz}\right.$, $\left.J_{15,16}=17.2 \mathrm{~Hz}, 1 \mathrm{H}, \mathrm{H}-15\right), 5.76\left(\mathrm{~d}, J_{1,2}=3.3 \mathrm{~Hz}, 1 \mathrm{H}, \mathrm{H}-1\right)$, $5.30\left(\mathrm{~d}, J_{16,15}=17.2 \mathrm{~Hz}, 1 \mathrm{H}, \mathrm{H}-16\right), 5.21\left(\mathrm{~d}, J_{16,15}=10.6 \mathrm{~Hz}\right.$, 1H, H-16), 4.79 (d, $J=11.1 \mathrm{~Hz}, 1 \mathrm{H}$, benzylic H), 4.77-4.71 (m, $\mathrm{H}-14,3 \mathrm{H}, 2 \times$ benzylic H), $4.63(\mathrm{~d}, J=11.8 \mathrm{~Hz}, 1 \mathrm{H}$, benzylic $\mathrm{H}), 4.62(\mathrm{~d}, J=11.4 \mathrm{~Hz}, 1 \mathrm{H}$, benzylic $\mathrm{H}), 4.56(\mathrm{~d}, J=11.9 \mathrm{~Hz}$, $1 \mathrm{H}$, benzylic $\mathrm{H}), 4.50(\mathrm{~d}, J=11.8 \mathrm{~Hz}, 1 \mathrm{H}$, benzylic $\mathrm{H}), 4.46$ (d, $J=10.8 \mathrm{~Hz}, 1 \mathrm{H}$, benzylic H), $4.43(\mathrm{~d}, J=11.8 \mathrm{~Hz}, 1 \mathrm{H}$, benzylic H), 4.42-4.37 (m, H-3', 4H, $3 \times$ benzylic H), 4.23 (dd, $J_{4}{ }^{\prime}, 5$, = $\left.5.9 \mathrm{~Hz}, J_{4}, 3^{\prime}=6.2 \mathrm{~Hz}, 1 \mathrm{H}, \mathrm{H}-4^{\prime}\right), 4.19$ (m, 1H, H-5), 4.07 (dd, $\left.J_{5^{\prime}, 6^{\prime}}=11.7 \mathrm{~Hz}, J_{5^{\prime}, 4^{\prime}}=5.9 \mathrm{~Hz}, 1 \mathrm{H}, \mathrm{H}-5^{\prime}\right), 3.96(\mathrm{~m}, 3 \mathrm{H}, 2 \times$ H-6', H-6), 3.83 (dd, $J_{1}$ ', ' = $10.3 \mathrm{~Hz}, 1 \mathrm{H}, \mathrm{H}-1^{\prime}$ ), 3.81 (m, 1H, H-3), $3.66\left(\mathrm{dd}, J_{5,6}=6.7 \mathrm{~Hz}, J_{6,6}=14.0 \mathrm{~Hz}, 1 \mathrm{H}, \mathrm{H}-6\right)$, 3.61-3.51 (m, 4H, $2 \times \mathrm{H}-11, \mathrm{H}-12, \mathrm{H}-1$ '), 3.45 (s, 3H, OMe), $3.43(\mathrm{dd}, J=2.25 \mathrm{~Hz}, J=7.2 \mathrm{~Hz}, 1 \mathrm{H}, \mathrm{H}-13), 3.39$ (s, $3 \mathrm{H}$, $\mathrm{OMe}), 3.38$ (m, 1H, H-2), 3.22 (s, 3H, OMe), 3.19 (m, 1H, $\mathrm{H}-4), 1.66$ (s, 3H, OAc), 1.05 (s, 9H, $t$-Bu) ppm; ${ }^{13} \mathrm{C}$ NMR $\delta$ $170.84(\mathrm{C}=\mathrm{O}), 156.83(\mathrm{C}-10), 138.73,138.68,138.42,138.36$, $138.24,137.88\left(6 \mathrm{C}_{\text {quat }}, 6 \times \mathrm{Ph}\right), 137.49(\mathrm{C}-7), 135.57(2 \mathrm{C}-\mathrm{Ph})$, 135.51 (2C-Ph), 134.97 (C-15), 133.44, 133.28 (2C quat, $2 \times \mathrm{Ph})$, $129.63(2 \mathrm{C}-\mathrm{Ph}), 129.58(2 \mathrm{C}-\mathrm{Ph}), 129.24(2 \times \mathrm{C}-9)$, 128.36-127.29 (m, 32C-Ar), 118.42 (C-16), 116.32 (2 × C-8), 105.17 (C-2'), 90.18 (C-1), 83.94 (C-3'), 83.59 (C-4'), 82.57 (C-13), 81.97 (C-5'), 81.47 (C-3), 80.01 (C-2), 79.85 (C-4), 78.66 (C-12), 77.21 (C-14), 75.41, 73.90, 73.41, 72.98, 72.35, $71.98(6 \times \mathrm{OBn}), 71.02(\mathrm{C}-11), 70.37(\mathrm{C}-1$ ') $), 69.51$ 
(C-5), 65.46 (C-6’), 61.10, 59.05, $59.05(3 \times \mathrm{OMe}), 50.50$ (C-6), 26.93 [3C, $\left.\mathrm{SiC}\left(\mathrm{CH}_{3}\right)_{3}\right], 22.90\left(\mathrm{CH}_{3} \mathrm{CO}_{2}\right), 19.27\left(\mathrm{C}_{\text {quat }}{ }^{-t}\right.$ $\mathrm{Bu}$ ) ppm; HRMS (ESI) $[\mathrm{M}+\mathrm{Na}]^{+}$calcd for $\mathrm{C}_{87} \mathrm{H}_{99} \mathrm{O}_{15} \mathrm{SiNa}$, 1448.6669; found, 1448.6682; anal. calcd for $\mathrm{C}_{87} \mathrm{H}_{99} \mathrm{NO}_{15} \mathrm{Si}$ (1426.83): C, 73.24; H, 6.99; N, 0.98; found: C, 73.23; H, 7.10; $\mathrm{N}, 1.08$.

\section{Synthesis of alcohol 22}

A solution of TBAF (113 mg, $0.43 \mathrm{mmol})$ in THF $(5 \mathrm{~mL})$ was added to a solution of compound 21 (205 $\mathrm{mg}, 0.14 \mathrm{mmol})$ in dry THF $(10 \mathrm{~mL})$, and the resulting mixture was stirred for $1 \mathrm{~h}$ at $\mathrm{rt}$, and concentrated. The crude product was purified by flash chromatography (hexanes-ethyl acetate, 50:50 to 40:60) to afford 22 (166 mg, $0.14 \mathrm{mmol}, 97 \%)$ as a colorless oil. TLC [hexanes-AcOEt (1:2)]: $R_{\mathrm{f}}=0.3 ;[\alpha]_{\mathrm{D}}{ }^{22}+27.3 ;{ }^{1} \mathrm{H}$ NMR $\delta$ $7.32-7.21(\mathrm{~m}, 30 \mathrm{H}, \mathrm{ArH}), 7.10\left(\mathrm{~d}, J_{9,8}=8.7 \mathrm{~Hz}, 2 \mathrm{H}, 2 \times \mathrm{H}-9\right)$, $6.84\left(\mathrm{~d}, J_{8,9}=8.7 \mathrm{~Hz}, 2 \mathrm{H}, 2 \times \mathrm{H}-8\right), 5.98\left(\mathrm{ddd}, J_{15,14}=6.0 \mathrm{~Hz}\right.$, $\left.J_{15,16}=10.4 \mathrm{~Hz}, J_{15,16}=17.3 \mathrm{~Hz}, 1 \mathrm{H}, \mathrm{H}-15\right), 5.52$ (d, $J_{1,2}=3.0$ $\mathrm{Hz}, 1 \mathrm{H}, \mathrm{H}-1), 5.36$ (d, $\left.J_{16,15}=17.3 \mathrm{~Hz}, 1 \mathrm{H}, \mathrm{H}-16\right), 5.28$ (d, $\left.J_{16,15}=10.4 \mathrm{~Hz}, 1 \mathrm{H}, \mathrm{H}-16\right), 4.81(\mathrm{~m}, \mathrm{H}-14,3 \mathrm{H}, 2 \times$ benzylic $\mathrm{H}), 4.70(\mathrm{~d}, J=11.6 \mathrm{~Hz}, 1 \mathrm{H}$, benzylic $\mathrm{H}), 4.66-4.57$ (m, $4 \mathrm{H}, 4$ $\times$ benzylic $\mathrm{H}), 4.52-4.48(\mathrm{~m}, 3 \mathrm{H}, 3 \times$ benzylic $\mathrm{H}), 4.42-4.38$ (m, 2H, H-3', benzylic H), 4.35 (d, $J=11.9 \mathrm{~Hz}, 1 \mathrm{H}$, benzylic H), 4.32-4.28 (m, 2H, H-5, H-4'), 4.02-3.95 (m, 3H, H-3, H-5', H-6), $3.85\left(\mathrm{dd}, J_{6,6}=14.1 \mathrm{~Hz}, J_{6,5}=5.8 \mathrm{~Hz}, 1 \mathrm{H}, \mathrm{H}-6\right)$, 3.77-3.72 (m, 2H, H-1',H-6'), 3.68-3.48 (m, 5H, $2 \times \mathrm{H} 11$, H-12, H-1', H-6'), 3.45 (m, 5H, H-2, H-13, OMe), 3.40 (s, 3H, OMe), 3.37 (br s, 1H, OH), 3.26 (m, 1H, H-4), 3.25 (s, 3H, OMe), 1.78 (s, 3H, OAc) ppm; ${ }^{13} \mathrm{C}$ NMR $\delta 171.83(\mathrm{C}=\mathrm{O})$, 156.89 (C-10), 138.54, 138.48, 138.39, 138.16, 138.09, 137.72 $\left(\mathrm{C}_{\text {quat }}, 6 \times \mathrm{Ph}\right), 137.46(\mathrm{C}-7), 135.01(\mathrm{C}-15), 129.18(2 \times \mathrm{C}-9)$, 127.40-128.37 (m, 30C-Ar), 118.47 (C-16), 116.31 (2 × C-8), 104.61 (C-2'), 90.64 (C-1), 83.71 (C-3'), 82.57 (C-13), 82.01 (C-5'), 81.46 (C-3), 81.23 (C-4'), 79.71 (C-2), 79.50 (C-4), 78.68 (C-12), 77.26 (C-14), 75.43, 74.10, 73.36, 72.78, 72.67, 72.53 (6 × OBn), 71.04 (C-11), 70.76 (C-1'), 70.15 (C-5), 62.23 (C-6'), 61.13, 59.07, 59.07 (3 × OMe), $50.15(\mathrm{C}-6), 22.93$ $\left(\underline{\mathrm{C}} \mathrm{H}_{3} \mathrm{CO}_{2}\right)$ ppm; HRMS (ESI) $[\mathrm{M}+\mathrm{Na}]^{+}$calcd for $\mathrm{C}_{71} \mathrm{H}_{81} \mathrm{NO}_{15} \mathrm{Na}, 1210.5504$; found, 1210.5544; anal. calcd for $\mathrm{C}_{71} \mathrm{H}_{81} \mathrm{NO}_{15}$ (1187.42): C, 71.76; H, 6.87; N, 1.18; found: $\mathrm{C}$, 71.93; H, 7.01; N, 1.08 .

\section{Synthesis of diolefin $\mathbf{2 4}$}

To a cooled solution $\left(-78{ }^{\circ} \mathrm{C}\right)$ of oxalyl chloride $(39 \mu \mathrm{L}$, $0.46 \mathrm{mmol})$ in DCM $(10 \mathrm{~mL})$, a solution of DMSO $(93 \mu \mathrm{L}$, $1.30 \mathrm{mmol})$ in DCM $(3 \mathrm{~mL})$ was added within $5 \mathrm{~min}$. After $10 \mathrm{~min}$ alcohol $22(155 \mathrm{mg}, 0.13 \mathrm{mmol})$ in DCM $(3 \mathrm{~mL})$ was added dropwise and stirring was continued for $10 \mathrm{~min}$ at $-78{ }^{\circ} \mathrm{C}$. Then, $\mathrm{Et}_{3} \mathrm{~N}(145 \mu \mathrm{L}, 1.04 \mathrm{mmol})$ was added and the mixture was allowed to attain $\mathrm{rt}$. Water $(7 \mathrm{~mL})$ was added, the organic layer was separated, dried, and concentrated. Crude aldehyde 23 was dissolved in DCM $(10 \mathrm{~mL})$ and this solution was added to a solution of amine $18(147 \mathrm{mg}, 0.52 \mathrm{mmol})$, acetic acid $(30 \mu \mathrm{L}, 0.52 \mathrm{mmol})$, and $\mathrm{MgSO}_{4}(\approx 200 \mathrm{mg})$ in DCM $(10 \mathrm{~mL})$, and the resulting mixture was stirred for $1 \mathrm{~h}$ at $\mathrm{rt}$. Then, $\mathrm{NaBH}_{3} \mathrm{CN}(12 \mathrm{mg}, 0.20 \mathrm{mmol})$ was added and the stirring was continued overnight. Water $(20 \mathrm{~mL}), 0.1 \mathrm{M}$ solution of $\mathrm{NH}_{3}(2 \mathrm{~mL})$, and DCM $(15 \mathrm{~mL})$ were added, the layers were separated, and the aqueous one extracted with DCM $(3 \times 10 \mathrm{~mL})$. Combined organic solutions were washed with water $(10 \mathrm{~mL})$ and brine $(10 \mathrm{~mL})$, dried, concentrated, and the residue was dissolved in 1,4-dioxane (10 mL). DMAP (3 mg, $0.03 \mathrm{mmol}), \mathrm{Et}_{3} \mathrm{~N}(91 \mu \mathrm{L}, 0.65 \mathrm{mmol})$, acetic anhydride $(37 \mu \mathrm{L}$, $0.39 \mathrm{mmol}$ ) were added, and the mixture was stirred overnight at $100{ }^{\circ} \mathrm{C}$. After cooling, water $(15 \mathrm{~mL})$ and DCM $(15 \mathrm{~mL})$ were added, the layers were separated, and the aqueous one was extracted with DCM $(3 \times 10 \mathrm{~mL})$. Combined organic solutions were washed with water $(10 \mathrm{~mL})$ and brine $(10 \mathrm{~mL})$, dried, concentrated, and the product was isolated by flash chromatography (hexanes-ethyl acetate, 25:75) to afford 24 (120 mg, $0.08 \mathrm{mmol}, 64 \%)$ as a colorless oil. TLC [hexanes-AcOEt $(1: 3)]: R_{\mathrm{f}}=0.25 ;[\alpha]_{\mathrm{D}}{ }^{22}+32.7 ;{ }^{1} \mathrm{H}$ NMR $\delta 7.25-7.06(\mathrm{~m}, 30 \mathrm{H}$, ArH), 7.06-6.95 (m, 4H, $\left.2 \times \mathrm{H}-9,2 \times \mathrm{H}_{-1}{ }^{\prime}\right), 6.79-6.72(\mathrm{~m}, 4 \mathrm{H}$, $2 \times \mathrm{H}-8,2 \times \mathrm{H}-8$ '), 5.91 (m, 1H, H-15), 5.82 (m, 1H, H-15'), $5.41\left(\mathrm{~d}, J_{1,2}=3.2 \mathrm{~Hz}, 1 \mathrm{H}, \mathrm{H}-1\right), 5.31-5.16(\mathrm{~m}, 4 \mathrm{H}, 2 \times \mathrm{H}-16,2$ $\times$ H-16'), $4.78(J=11.1 \mathrm{~Hz}, 1 \mathrm{H}$, benzylic $\mathrm{H}), 4.75-4.69(\mathrm{~m}$, $2 \mathrm{H}$, benzylic $\mathrm{H}, \mathrm{H}-14), 4.57-4.45(\mathrm{~m}, 4 \mathrm{H}, 4 \times$ benzylic $\mathrm{H})$, 4.43-4.36 (m, 3H, $2 \times$ benzylic H, H-12'), 4.35-4.26 (m, 3H, 2 $\times$ benzylic H, H-4'), $4.18(\mathrm{~m}, 1 \mathrm{H}, \mathrm{H}-5), 4.12\left(\mathrm{dd}, J_{11}{ }^{\prime}, 11^{\prime}\right.$ ' = $10.0 \mathrm{~Hz}, J_{11^{\prime}, 12}$, = $2.1 \mathrm{~Hz}, 1 \mathrm{H}, \mathrm{H}-11^{\prime}$ ), 4.06-4.02 (m, 2H, H-5', H-3'), 3.94-3.82 (m, 4H, $2 \times$ H-6, H-6', H-11'), 3.79-3.70 (m, 3H, H-6', H-14', H-11,), 3.63-3.56 (3H, m, H-3, H-11, H-12), 3.54-3.43 (m, 4H, $2 \times \mathrm{H}^{-1}$ ', H-13, H-13'), 3.40 (s, 3H, OMe), 3.37 (s, 3H, OMe), 3.33-3.28 (m, 7H, $2 \times \mathrm{OMe}, \mathrm{H}-2), 3.25$ (s, 3H, OMe), 3.21 (m, 1H, H-4), 3.16 (s, 3H, OMe), 1.67 (s, 3H, OAc), 1.64 (s, 3H, OAc) ppm; ${ }^{13} \mathrm{C}$ NMR $\delta 171.13$, $170.77(2 \times \mathrm{C}=\mathrm{O}), 157.98,156.79$ (C-10, C-10'), 138.97, 138.79, 138.46, 138.32, 138.32, 137.99, 137.89, 136.04 (Cquat, $\left.6 \times \mathrm{Ph}, \mathrm{C}-7, \mathrm{C}-7^{\prime}\right), 135.74$ (C-15'), 135.09 (C-15), 129.52, $\left.129.44\left(2 \times \mathrm{C}-9,2 \times \mathrm{C}^{-9}\right)^{\prime}\right), 128.31-127.25$ (m, $\left.30 \times \mathrm{C}-\mathrm{Ar}\right)$, 118.48, 118.43 (C-16, C-16'), 116.23, $115.18(2 \times \mathrm{C}-8$, $\left.2 \times \mathrm{C}^{-8}{ }^{\prime}\right), 104.93$ (C-2'), 89.27 (C-1), 84.05 (C-3'), 83.54 (C-4'), 82.56 (C-13), 81.81 (C-14'), 81.73 (C-12'), 80.13 (C-2), 79.75 (C-13'), 79.23 (C-4), 78.81 (C-3), 78.73 (C-12), 77.54 (C-14), 77.48 (C-5'), 75.22, 73.95, 73.26, 72.67, 72.27, $72.25(6 \times \mathrm{OBn}), 71.16(\mathrm{C}-11), 71.04\left(\mathrm{C}-1^{\prime}\right), 70.13(\mathrm{C}-5)$, 66.55 (C-11'), 61.20, 61.10, 59.05, 59.01, 58.24, 56.70 $(6 \times \mathrm{OMe}), 52.08\left(\mathrm{C}^{\prime} 6^{\prime}\right), 49.66(\mathrm{C}-6), 22.90,22.75(2 \times$ $\mathrm{CH}_{3} \mathrm{CO}_{2}$ ) ppm; HRMS (ESI) $[\mathrm{M}+\mathrm{Na}]^{+} \mathrm{calcd}$ for $\mathrm{C}_{88} \mathrm{H}_{104} \mathrm{~N}_{2} \mathrm{O}_{19} \mathrm{Na}$, 1515.7153; found, 1515.7131; anal. calcd for $\mathrm{C}_{88} \mathrm{H}_{104} \mathrm{~N}_{2} \mathrm{O}_{19}(1493.80)$ : C, 70.76; $\mathrm{H}, 7.02 ; \mathrm{N}, 1.88$; found: C, 70.62; H, 7.10; N, 1.90 . 


\section{Synthesis of macrocyclic compound $\mathbf{2 5}$}

To a solution of diene $24(85.0 \mathrm{mg}, 0.060 \mathrm{mmol})$ in degassed, anhydrous toluene $(10 \mathrm{~mL})$, Hoveyda-Grubbs catalyst 2 nd generation $(3.7 \mathrm{mg}, 0.006 \mathrm{mmol})$ was added, and the mixture was stirred and heated at $95{ }^{\circ} \mathrm{C}$ for $48 \mathrm{~h}$. The mixture was concentrated and the product was purified by flash chromatography (hexanes-ethyl acetate, 15:85) to give macrocycle 25 (22.8 mg, $0.016 \mathrm{mmol}, 26 \%)$ as a white amorphous foam. TLC [hexanes-AcOEt (1:5)]: $R_{\mathrm{f}}=0.2 ;[\alpha]_{\mathrm{D}}{ }^{22}+13.2 ;{ }^{1} \mathrm{H}$ NMR $\delta$ 7.40-7.02 (m, 36H, ArH), 6.60 (m, 2H, ArH), 5.97 (dd, $J_{15}, 15$ $\left.=15.9 \mathrm{~Hz}, J_{15}, 14^{\prime}=6.4 \mathrm{~Hz}, 1 \mathrm{H}, \mathrm{H}-15^{\prime}\right), 5.74-5.68(\mathrm{~m}, 2 \mathrm{H}$, H-1, H-15), 4.87 (m, 1H, H-14'), 4.70 (d, $J=12.0 \mathrm{~Hz}, 1 \mathrm{H}$, benzylic H), 4.66 (m, 1H, H-5'), 4.59 (d, $J=10.8 \mathrm{~Hz}, 1 \mathrm{H}$, benzylic $\mathrm{H}), 4.58(\mathrm{~d}, J=11.7 \mathrm{~Hz}, 1 \mathrm{H}$, benzylic $\mathrm{H}), 4.54(\mathrm{~d}, J=$ $10.8 \mathrm{~Hz}, 1 \mathrm{H}$, benzylic $\mathrm{H}), 4.50$ (d, $J=11.2 \mathrm{~Hz}, 1 \mathrm{H}$, benzylic $\mathrm{H})$, $4.48(\mathrm{~d}, J=11.7 \mathrm{~Hz}, 1 \mathrm{H}$, benzylic H), 4.48 (d, $J=11.7 \mathrm{~Hz}, 1 \mathrm{H}$, benzylic H), 4.46 (dd, $J=7.3 \mathrm{~Hz}, J=6.0 \mathrm{~Hz}, 1 \mathrm{H}, \mathrm{H}-4$ '), 4.41-4.33 (m, 4H, $3 \times$ benzylic H, H-6'), 4.30 (d, $J=11.0 \mathrm{~Hz}$, $1 \mathrm{H}$, benzylic $\mathrm{H}), 4.15(\mathrm{~d}, J=10.8 \mathrm{~Hz}, 1 \mathrm{H}$, benzylic $\mathrm{H})$, 4.02-3.96 (m, 2H, H-6, H-3'), 3.95-3.90 (m, 2H, H-1', H-14), 3.82 (m, 1H, H-5), 3.77 (m, 1H, H-11'), 3.72-3.61 (5H, H-12, H-12', H-1', H-11, H-11'), 3.58 (m, 1H, H-11), 3.55-3.51 (m, 2H, H-13, H-13'), 3.50 (s, 3H, OMe), 3.49 (s, 3H, OMe), 3.41 (s, 3H, OMe), 3.41 (s, 3H, OMe), 3.39 (s, 3H, OMe), 3.37 (s, $3 \mathrm{H}, \mathrm{OMe}), 3.35$ (m, 1H, H-6'), $3.23\left(\mathrm{dd}, J_{2,3}=6.7 \mathrm{~Hz}, J_{2,1}=\right.$ $4.0 \mathrm{~Hz}, 1 \mathrm{H}, \mathrm{H}-2), 3.14$ (dd, $\left.J_{3,4}=9.5 \mathrm{~Hz}, 1 \mathrm{H}, \mathrm{H}-3\right), 3.07(\mathrm{~m}$, $1 \mathrm{H}, \mathrm{H}-6), 3.00$ (dd, $\left.J_{4,5}=9.0 \mathrm{~Hz}, 1 \mathrm{H}, \mathrm{H}-4\right), 1.90$ (s, 3H, OAc), 1.73 (s, 3H, OAc) ppm; ${ }^{13} \mathrm{C} \mathrm{NMR}(125 \mathrm{MHz}) \delta 171.21,170.78$ $(2 \times \mathrm{C}=\mathrm{O}), 157.56,157.49$ (C-10, C-10'), 138.50, 138.50, $138.39,138.13,138.00,137.83,137.53,137.36\left(\mathrm{C}_{\text {quat }}, 6 \times \mathrm{Ph}\right.$, C-7, C-7'), 131.73 (C-15'), 130.50 (C-15), 129.46, 129.02 (2× C-9, $2 \times$ C-9'), 128.98-128.41 (m, $30 \times$ C-Ar), 119.31, 115.03 ( $\left.2 \times \mathrm{C}-8,2 \times \mathrm{C}^{8} 8^{\prime}\right), 105.77$ (C-2'), 91.08 (C-1), 84.23 (C-3'), 84.23 (C-4'), 83.73 (C-13), 82.10 (C-13'), 81.79 (C-3), 81.27 (C-14), 79.68 (C-12'), 79.41 (C-5'), 79.41 (C-2), 78.95 (C-14'), 78.32 (C-12), 76.75 (C-4), 75.65, 73.72, 73.43, 73.28, 72.39, 72.23 (6 × OBn), 70.69 (C-11), 70.56 (C-5), 69.05 (C-1'), 67.27 (C-11'), 61.22, 59.54, 59.12, 59.01, 58.66, 57.07 (6 × OMe), 56.43 (C-6'), 49.57 (C-6), 23.06, $22.68\left(2 \times \mathrm{CH}_{3} \mathrm{CO}_{2}\right) \mathrm{ppm}$; HRMS (ESI) $[\mathrm{M}+\mathrm{Na}]^{+}$calcd for $\mathrm{C}_{86} \mathrm{H}_{100} \mathrm{~N}_{2} \mathrm{O}_{19} \mathrm{Na}$, 1487.6857; found, 1487.6971; anal. calcd for $\mathrm{C}_{86} \mathrm{H}_{100} \mathrm{~N}_{2} \mathrm{O}_{19}$ (1465.74): C, 70.47; H, 6.88; N, 1.91; found: C, 70.62; H, 6.96; $\mathrm{N}, 1.84$.

\section{Supporting Information}

\section{Supporting Information File 1}

Copies of NMR spectra.

[https://www.beilstein-journals.org/bjoc/content/

supplementary/1860-5397-14-50-S1.pdf]

\section{Acknowledgements}

The support from the Grant UMO-2012/05/B/ST5/00377 from National Science Centre is gratefully acknowledged.

\section{ORCID ${ }^{\circledR}$ iDs}

Karolina Tiara - https://orcid.org/0000-0001-5726-6887 Mykhaylo A. Potopnyk - https://orcid.org/0000-0002-4543-2785

Sławomir Jarosz - https://orcid.org/0000-0002-9212-6203

\section{References}

1. Zhang, X.; Yin, J.; Yoon, J. Chem. Rev. 2014, 114, 4918-4959. doi:10.1021/cr400568b

2. Kolesnichenko, I. V.; Anslyn, E. V. Chem. Soc. Rev. 2017, 46, 2385-2390. doi:10.1039/C7CS00078B

3. Amato, M. E.; Ballistreri, F. P.; Gentile, S.; Pappalardo, A.; Tomaselli, G. A.; Toscano, R. M. J. Org. Chem. 2010, 75, 1437-1443. doi:10.1021/jo902328y

4. Akdeniz, A.; Minami, T.; Watanabe, S.; Yokoyama, M.; Ema, T.; Anzenbacher, P., Jr. Chem. Sci. 2016, 7, 2016-2022. doi:10.1039/C5SC04235F

5. McConnell, A. J.; Beer, P. D. Angew. Chem., Int. Ed. 2012, 51, 5052-5061. doi:10.1002/anie.201107244

6. Ema, T.; Ura, N.; Eguchi, K.; Ise, Y.; Sakai, T. Chem. Commun. 2011, 47, 6090-6092. doi:10.1039/C1CC11572C

7. Caricato, M.; Leza, N. J.; Gargiulli, C.; Gattuso, G.; Dondi, D.; Pasini, D. Beilstein J. Org. Chem. 2012, 8, 967-976. doi:10.3762/bjoc.8.109

8. Pu, L. Acc. Chem. Res. 2012, 45, 150-163. doi:10.1021/ar200048d

9. Ema, T.; Yokoyama, M.; Watanabe, S.; Sasaki, S.; Ota, H.; Takaishi, K. Org. Lett. 2017, 19, 4070-4073. doi:10.1021/acs.orglett.7b01838

10. Brea, R. J.; Reiriz, C.; Granja, J. R. Chem. Soc. Rev. 2010, 39, 1448-1456. doi:10.1039/B805753M

11. Quinn, T. P.; Atwood, P. D.; Tanski, J. M.; Moore, T. F.; Folmer-Andersen, J. F. J. Org. Chem. 2011, 76, 10020-10030. doi:10.1021/jo2018203

12. Šolomek, T.; Powers-Riggs, N. E.; Wu, Y.-L.; Young, R. M.; Krzyaniak, M. D.; Horwitz, N. E.; Wasielewski, M. R. J. Am. Chem. Soc. 2017, 139, 3348-3351. doi:10.1021/jacs.7b00233

13. Potopnyk, M. A.; Jarosz, S. Adv. Carbohydr. Chem. Biochem. 2014, 71, 227-295. doi:10.1016/B978-0-12-800128-8.00003-0

14. Jarosz, S.; Potopnyk, M. A.; Kowalski, M. Carbohydr. Chem. 2014, 40, 236-256. doi:10.1039/9781849739986-00236

15. Potopnyk, M. A.; Jarosz, S. "Sweet" Sucrose Macrocycles via a "Click Chemistry” Route. In Click Chemistry in Glycoscience; Witczak, Z. J.; Bielski, R., Eds.; Wiley, 2013; pp 235-250. doi:10.1002/9781118526996.ch9

16. Jarosz, S.; Listkowski, A.; Lewandowski, B.; Ciunik, Z.; Brzuszkiewicz, A. Tetrahedron 2005, 61, 8485-8492. doi:10.1016/j.tet.2005.06.046

17. Potopnyk, M. A.; Lewandowski, B.; Jarosz, S. Tetrahedron: Asymmetry 2012, 23, 1474-1479. doi:10.1016/j.tetasy.2012.10.003

18. Potopnyk, M. A.; Jarosz, S. Eur. J. Org. Chem. 2013, 5117-5126. doi:10.1002/ejoc.201300427

19. Potopnyk, M. A.; Cmoch, P.; Jarosz, S. Org. Lett. 2012, 14, 4258-4261. doi:10.1021/ol301993d

20. Potopnyk, M. A.; Jarosz, S. Monatsh. Chem. 2013, 144, 437-443. doi:10.1007/s00706-012-0894-2 
21. Łęczycka-Wilk, K.; Dąbrowa, K.; Cmoch, P.; Jarosz, S. Org. Lett. 2017, 19, 4596-4599. doi:10.1021/acs.orglett.7b02198

22. Barros, M. T.; Petrova, K. T.; Singh, R. P. Eur. Polym. J. 2010, 46, 1151-1157. doi:10.1016/j.eurpolymj.2010.02.002

23. Petrova, K. T.; Potewar, T. M.; Ascenso, O. S.; Barros, M. T. Carbohydr. Polym. 2014, 110, 38-46. doi:10.1016/j.carbpol.2014.03.050

24. Petrova, K. T.; Correia-da-Silva, P.; Crucho, C. I. C.; Barros, M. T. Curr. Org. Chem. 2014, 18, 1788-1802. doi:10.2174/1385272819666140527231535

25. Crucho, C. I. C.; Barros, M. T. J. Mater. Chem. B 2014, 2, 3946-3955. doi:10.1039/C3TB21632B

26. Raposo, C. D.; Petrova, K. T.; Barros, M. T.; Calhelha, R. C.; Soković, M.; Ferreira, I. C. F. R. Med. Chem. 2016, 12, 22-29. doi:10.2174/1573406410666150807111029

27. Petrova, K. T.; Barros, M. T.; Calhelha, R. C.; Soković, C.; Ferreira, I. C. F. R. Med. Chem. Res. 2018, 27, 980-988. doi:10.1007/s00044-017-2121-5

28. Łęczycka, K.; Jarosz, S. Tetrahedron 2015, 71, 9216-9222. doi:10.1016/j.tet.2015.10.046

29. Wakchaure, S.; Einsiedel, J.; Waibel, R.; Gmeiner, P. Synthesis 2012, 44, 2682-2694. doi:10.1055/s-0032-1316758

30. Mao, Z.-Y.; Si, C.-M.; Liu, Y.-W.; Dong, H.-Q.; Wei, B.-G.; Lin, G.-Q. J. Org. Chem. 2017, 82, 10830-10845. doi:10.1021/acs.joc.7b01598

31. Bernet, B.; Vasella, A. Helv. Chim. Acta 1979, 62, 1990-2016. doi:10.1002/hlca.19790620629

32. Bernet, B.; Vasella, A. Helv. Chim. Acta 1979, 62, 2400-2410. doi:10.1002/hlca.19790620736

33. Jensen, T.; Mikkelsen, M.; Lauritsen, A.; Andresen, T. L.; Gotfredsen, C. H.; Madsen, R. J. Org. Chem. 2009, 74, 8886-8889. doi:10.1021/j09019495

34. Fürstner, A.; Weidmann, H. J. Org. Chem. 1989, 54, 2307-2311. doi:10.1021/jo00271a012

35. Fürstner, A.; Weidmann, H. J. Org. Chem. 1990, 55, 1363-1366. doi:10.1021/jo00291a053

36. Gaweł, A.; Jarosz, S. J. Carbohydr. Chem. 2010, 29, 332-347. doi:10.1080/07328303.2010.524958

37. Szyszka, Ł.; Osuch-Kwiatkowska, A.; Potopnyk, M. A.; Jarosz, S. Beilstein J. Org. Chem. 2017, 13, 2146-2152. doi:10.3762/bjoc.13.213

38. Mancuso, A. J.; Huang, S.-L.; Swern, D. J. Org. Chem. 1978, 43, 2480-2482. doi:10.1021/jo00406a041

\section{License and Terms}

This is an Open Access article under the terms of the Creative Commons Attribution License

(http://creativecommons.org/licenses/by/4.0), which permits unrestricted use, distribution, and reproduction in any medium, provided the original work is properly cited.

The license is subject to the Beilstein Journal of Organic Chemistry terms and conditions:

(https://www.beilstein-journals.org/bjoc)

The definitive version of this article is the electronic one which can be found at:

doi:10.3762/bjoc. 14.50 